\title{
Producción de cursos en-línea basados en objetos de aprendizaje: una propuesta metodológica orientada a estudiantes de carreras técnicas
}

\section{Production of on-line courses based on learning objects: a methodological proposal aimed at students of technical careers}

\author{
Margarita Elizabeth Zambrano ${ }^{1}$, Walter Marcelo Fuertes-Diaz ${ }^{1}$, Diego David Pérez-Herrera ${ }^{1}$, César Javier \\ Villacís ${ }^{1}$, Ernesto Pérez-Estévez ${ }^{2}$ \\ ${ }^{1}$ Universidad de las Fuerzas Armadas - ESPE, Sangolquí, Ecuador \\ ${ }^{2}$ Consorcio Ecuatoriano para el Desarrollo de Internet Avanzado - CEDIA, Cuenca, Ecuador
}

Correo a/Mail to: Walter Marcelo Fuertes-Diaz (wmfuertes@espe.edu.ec)

Agradecimientos/Acknowledgments: PhD. Jenny Gabriela Torres Olmedo, Escuela Politécnica Nacional, Quito, Ecuador; PhD. Luis Enrique Sánchez Crespo, Universidad Castilla La Mancha, España; y Mag. Mario Bernabé Ron, Universidad de las Fuerzas Armadas - ESPE, Sangolquí, Ecuador por su aporte invaluable como miembros del Proyecto de Investigación referido.

Financiamiento/Funding: Este trabajo ha sido parcialmente financiado en el marco del proyecto de investigación titulado "Plataformas Computacionales de Entrenamiento, Experimentación, Gestión y Mitigación de Ataques a la Ciberseguridad", Código: ESPE-2015-PIC-019, Fase 1 con el cofinanciamiento del Consorcio Ecuatoriano para el Desarrollo de Internet Avanzado (CEDIA).

\section{Recibido/Submitted: 21 Dez. 2016; Aceptado/Approved: 21 Dez. 2016}

Copyright (c) 2016 Zambrano et al.. Todo el contenido de la revista (incluyendo instrucciones, política editorial y modelos) está bajo una licencia Creative Commons Atribución-NoComercial-Compartirlgual 3.0 No Adaptada. Siendo publicados por esta revista, artículos son de libre uso en ambientes educativos, de investigación y no comerciales, con atribución de autoría obligatoria. Más información en http://ojs.c3sl.ufpr.br/ojs2/index.php/atoz/about/submissions\#copyrightNotice.

\begin{abstract}
Resumen
Introducción: Este estudio tiene como objetivo estimular la comprensión conceptual y procedural de estudiantes de carreras técnicas a través de una propuesta metodológica que se aplica durante el diseño e implementación de Cursos en-línea basados en objetos de aprendizaje (OA). Método: Se han aplicado de manera armonizada el método de diseño de hipermedia orientado a objetos (OOHDM) y la metodología para la creación de OA (CROA) para la producción de un curso de Seguridad de la Información, como prueba de concepto. El curso fue desarrollado bajo licencia académicaArticulate Studio para contenidos y evaluaciones, y con Ardora para la elaboración de actividades de aprendizaje. Ambos fueron implantados dentro de Moodle como entorno virtual de E/A (EVEA). Para las instancias de auto evaluación y reflexión, se aplicó el aprendizaje basado en problemas (ABP), que además promueve experiencias colaborativas entre estudiantes y aprendientes. Para la evaluación de la usabilidad del curso, se aplicó la matriz para la evaluación de OA LORI.

Resultados: Los resultados muestran, por un lado el procedimiento metodológico teórico y práctico validado, y por otro, muestran la satisfacción de los estudiantes con respecto a la organización, contenido y funcionalidad del curso.

Conclusión: La aplicación conjunta de las metodologías OOHDM, CROA y ABP, permiten estructurar sistemáticamente y pedagógicamente los materiales educativos en base a OA de cursos en línea, para mejorar la comprensión conceptual y procedural de los estudiantes de carreras técnicas.
\end{abstract}

Palabras clave: Objetos de aprendizaje (OA); Metodología de criação de objetos de aprendizagem (CROA); Diseño Instruccional; Entorno Virtual de Enseñanza-Aprendizaje (EVA); Aprendizaje Basado en Problemas (ABP); método de diseño de hipermedia orientado a objetos (OOHDM)

\begin{abstract}
Introduction: This research main aim has been to stimulate the conceptual and procedural understanding of students of technical careers through a methodology that is applied during the design and implementation of online courses, which are based on Learning Objects.

Method: We applied a harmonized Object-Oriented Hypermedia Design Method (OOHDM) and the methodology for the Creation of Learning Objects (CROA) for the production of a course in Information Security as proof of the concept. The course has been developed with an academic license of Articulate Studio for the content and assessments as well as with Ardora for developing learning activities. Both of them were introduced within Moodle as virtual teaching and learning environment (VLE). For cases of self-assessment and reflection, the Learning Based on Problems (ABP) has been applied, which also promotes collaborative experiences between students and learners. For the evaluation of the usability of the course, the LORI matrix for the evaluation of learning objects we have used.

Results: The results demonstrate firstly the theoretical and practical validated methodological procedure, and secondly, they indicate the student's satisfaction regarding the organization, content and functionality of the course.

Conclusio: $n$ : The blend application of the OOHDM, CROA and LBP methodologies allow to structure systematically and pedagogically educational materials, which are based on Learning Objects for online courses to improve conceptual and procedural comprehension in students of technical careers.

Keywords: Learning objects (OA); Methodology of creation of learning objects (CROA); Instructional design; Object Oriented Hypermidia Design Model (OOHDM); Virtual Learning Environment (AVA); Problem Based Learning (PBL)
\end{abstract}

\section{INTRODUCCIÓN}

En los últimos años la comunidad académica y científica se ha preocupado por innovar los entornos virtuales de enseñanza-aprendizaje (EVEA) en la Educación Superior incorporando la aplicación de Objetos de Aprendizaje 
(OA). Algunas investigaciones como García Vera, Roig Vila, Marti Ciriquian, y García Ferrández (2012) describen el uso de OA para cursos de asignaturas técnicas. Maldonado, Carvallo, y Siguencia (2015), presentan un estudio comparativo de propuestas metodológicas para el diseño de OA a nivel Iberoamericano, donde se destaca CROA. Soto de Giorgis, Palma Muñoz, y Roncagliolo de la Horra (2002), explican los modelos de diseño conceptual, de contexto y el modelo navegacional de cursos en línea para EVEAs. De Giusti et al. (2015), explican los resultados preliminares de la utilización de OA para la enseñanza universitaria, aplicando el diseño instruccional alineado con la metodología CROA. Alonso, Castillo, Martínez, y Muñoz (2013), presentan la Metodología MEDOA para el desarrollo de OA que es una metodología similar a CROA. Mestre, Torres, Díaz, y Gómez (2014), exponen los lineamientos pedagógicos, comunicativos y tecnológicos para la producción de cursos mediados por Tics en la Educación a Distancia.

A pesar de estos esfuerzos, en estos trabajos no se distingue los lineamientos metodológicos para el diseño de cursos en línea en base a principios de Ingeniería de Software para garantizar un material digital didáctico reutilizable. Además no se especifican los procedimientos de diseño, desarrollo, implantación y almacenamiento de los OA, ni tampoco contempla una licencia de autor para el uso comercial o académico de dicha metodología.

Ante este panorama, este estudio intenta aportar con una propuesta metodológica que coadyuve a estimular la capacidad de comprender o aprehender (i. e. traducción, interpretación y extrapolación, según la Taxonomía de Bloom). En consecuencia, nuestra premisa de investigación a resolver sería: ¿En qué medida el diseño y desarrollo de cursos on-line con OA combinados con aprendizaje basado en problemas (ABP), mejoraría los niveles de comprensión que poseen los estudiantes del 5 to nivel de una carrera de ingeniería?

Este estudio tiene como objetivo estimular la comprensión conceptual y procedural de los estudiantes a través de una propuesta metodológica que se aplica sistemáticamente durante el diseño e implementación de Cursos On-Line basados en OA. Para llevarlo a cabo, se han aplicado de manera armonizada el Método de Diseño de Hipermedia Orientado a Objetos (OOHDM) (Pressman, 2010) para el diseño navegacional del sitio Web del curso y el diseño conceptual de la aplicación; y la metodología para la creación de OA (CROA) (Sanz, Moralejo, \& Barranquero, 2014). Esto mantiene una apropiada organización de los contenidos del curso y permite reducir contenidos extensos en pequeñas unidades pedagógicas compactas e interactivas.

Como prueba de concepto, se implementaron los OA del curso de Fundamentos de Seguridad de la Información, que fueron desarrollados bajo licencia académica Articulate Studio para contenidos y evaluaciones, donde también se utilizó Ardora para la elaboración de actividades de aprendizaje. Estos fueron integrados mediante Reload para formar un paquete SCORM, que puede ser implantado dentro de la plataforma Moodle, que es un entorno virtual de distribución gratuita en donde se pueden llevar a cabo varios procesos pedagógicos sobre la Web. Para las instancias de auto evaluación y reflexión, se aplicó el ABP que promueve experiencias colaborativas entre estudiantes y aprendientes. Para la evaluación de la usabilidad del curso, se aplicó la matriz de evaluación de OA LORI. Los resultados muestran que esta integración permite estructurar los materiales educativos en base a OA de los cursos en línea, que mejoran la comprensión conceptual y procedural de los estudiantes de quinto nivel de carreras de ingeniería, población objeto de este estudio.

\section{PROCEDIMIENTOS METODOLÓGICOS Y RESULTADOS}

Desde el punto de vista académico, esta propuesta está fundamentada en tres pilares: i) El Constructivismo, como modelo educativo que implica generar estrategias de aprendizaje para desarrollar las competencias de un estudiante, no solo en lo referente a conocimientos sino también a destrezas, habilidades, actitudes y valores; ii) La adquisición de mayores conocimientos estimulando la comprensión. Es decir desarrollar en los educandos el pensamiento crítico, el análisis de texto, y el uso de las herramientas informáticas para facilitar el proceso de adquisición de conocimientos; y iii) El desarrollo de entornos virtuales de enseñanza-aprendizaje, también conocidos como sistemas de gestión de conocimiento (LMS), que posibilitan la interactividad entre profesores y estudiantes fomentando el aprendizaje colaborativo. La articulación de estos tres pilares permite lograr en los estudiantes efectos satisfactorios en su aprendizaje (figura 1).

Tal como se puede apreciar en la figura 1, esta propuesta está dividida en dos grandes bloques. El primero que tiene que ver con el proceso ingenieril de desarrollo e implantación del software a través de OOHDM. El segundo concerniente a la creación de OA con la Metodología CROA, con cada una de sus etapas. Esto ha sido articulado con la técnica didáctica de aprendizaje basado en problemas, para desarrollar en los estudiantes las habilidades de comunicación, trabajo colaborativo, investigación, selección de información y auto-aprendizaje. Finalmente, muestra cómo se vincula la etapa de Diseño con la Taxonomía de Bloom, para evaluar dos de sus dominios: a) el conocimiento, que es la información, los principios y las ideas sobre un tema específico; b) la comprensión, que es la capacidad que desarrollan los estudiantes para entender, explicar, describir, comparar y distinguir objetos del mundo real. De esta propuesta se han derivado una guía metodológica y un curso en-línea de Fundamentos de Seguridad de la Información. A continuación se describe brevemente las fases de la propuesta: 


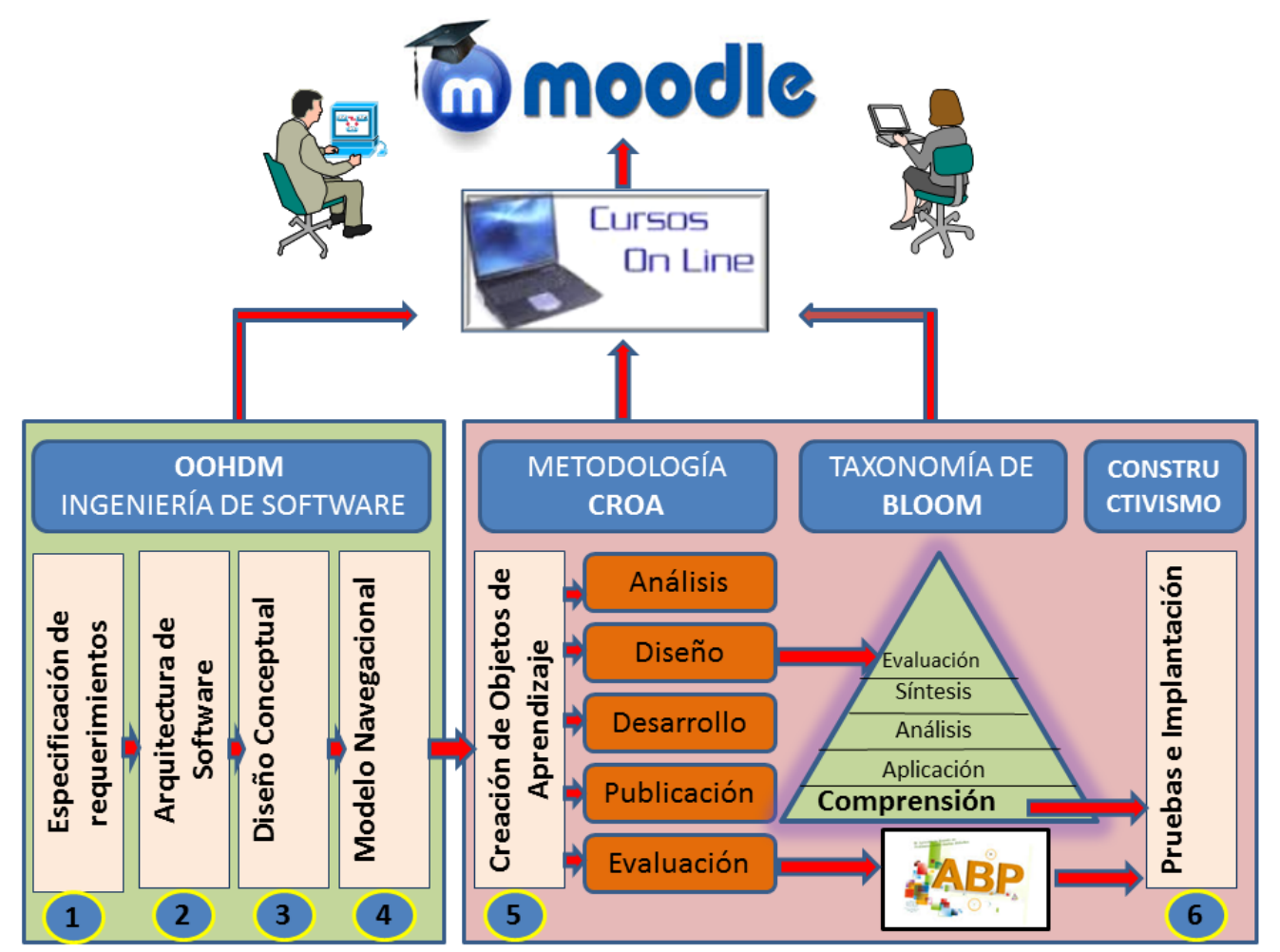

Figura 1. Elementos de la propuesta metodológica y sus interrelaciones.

Fuente: los autores.

\section{Fase 1: Especificación de Requerimientos}

Como requisitos esenciales (conforme al estándar IEEE-830, 1998), el curso en-línea de Fundamentos de Seguridad de la Información debía tener al menos tres unidades didácticas: (1) Programas indeseables o maliciosos; (2) Tipos de ataques de Ciberseguridad; (3) Análisis de vulnerabilidades. Debía ser subido a un entorno virtual de E/A, por lo que fue subido en Moodle, en un aula virtual de CEDIA (Red Nacional de Educación e Investigación del Ecuador). Debía incluir actividades de aprendizaje individuales y colaborativas aplicando el aprendizaje basado en problemas. Debía vincular las interrelaciones de sus dos actores principales, el estudiante autenticado y el tutor. El primero, que cumpliría las siguientes tareas y roles: (i) Acceder al aula virtual; (ii) Acceder a los materiales del curso; (iii) Acceder a las lecciones del curso; (iv) Acceder a los OA del curso; (v) Interactuar con herramientas de colaboración; (vi) Revisar los problemas resueltos (i.e., revisar los objetivos, acceder a los contenidos, resolver las actividades de aprendizaje y el test de auto-evaluación); (vii) Resolver los problemas propuestos. El segundo actor, por su parte realizaría el seguimiento académico, revisaría los foros y verificaría la resolución de los problemas propuestos.

\section{Fase 2: Arquitectura de Software}

El curso en-línea ha sido diseñado con una arquitectura Cliente/Servidor en n-capas las cuales son: a) Capa del Usuario, que le permite navegar por el sitio Web; b) Capa del LMS, que permite acceder a los cursos de formación y capacitación que ofrece CEDIA, a la bases de datos de Moodle y a los OA que tiene cada uno de los tres cursos desarrollados.

\section{Fase 3: Diseño Conceptual}

En esta fase se generó el modelo conceptual de la aplicación, donde las clases, relaciones y cardinalidades se definen de acuerdo a las reglas que se aplican sobre los Diagramas de Interacción de Usuario. Este modelo ha considerado las entidades que se relacionan dentro de Moodle con los OA del curso, donde se distinguen metadatos de tipo educacional.

\section{Fase 4: Modelo Navegacional}

Una vez realizado el diseño conceptual, hay que diseñar el Modelo Navegacional, que es una vista del modelo conceptual que refleja la información accesible a un usuario, y los caminos y estructuras de acceso para llegar a ella. De modo que, en esta propuesta se deben diseñar los aspectos navegacionales, teniendo en cuenta qué objetos del modelo básico van a ser navegables, qué tipo de relaciones, estructuras de composición y conexiones 
existen entre los objetos. Esto permite a la aplicación Web ejecutar todas las tareas requeridas por los actores. En este estudio, se han considerado el uso de formularios Web de Moodle y los OA que han sido generados con Articulate Studio y Ardora.

\section{Fase 5: Creación de OA}

De acuerdo con Sanz et al. (2014), CROA es una metodología empleada para la creación de OA basada en el Diseño Instruccional, compuesta por cinco fases: Análisis, Diseño, Desarrollo, Publicación, y Evaluación. La figura 2 muestra que en cada fase existen varios insumos, técnicas, herramientas y grupos de documentos que cumplen con los principios del Diseño Instruccional y la Taxonomía de Bloom. Los OA del presente proyecto de investigación fueron diseñados y desarrollados en base a esta metodología. En esta fase de nuestra propuesta se describirán las cinco etapas:

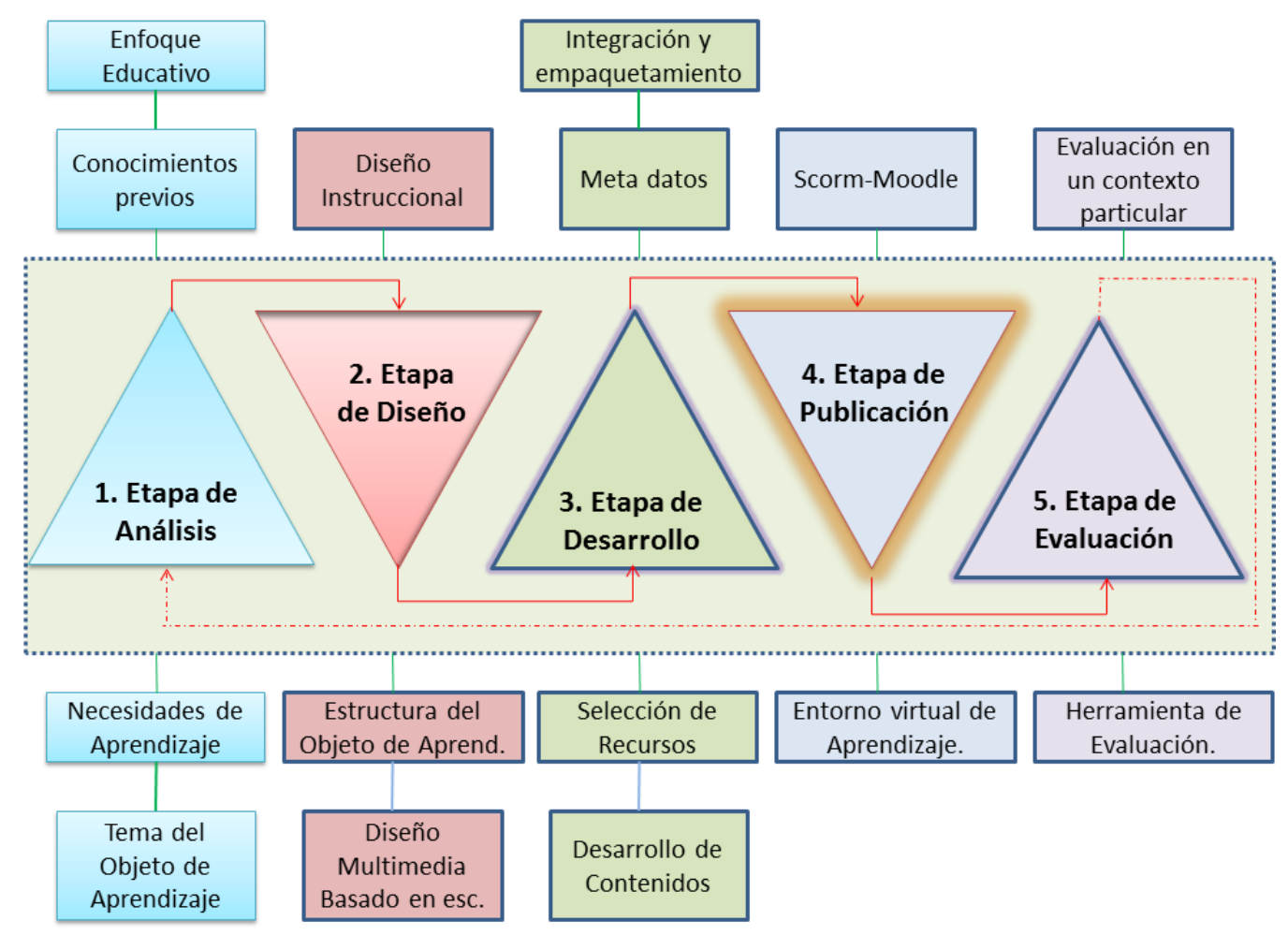

Figura 2. Fases de la Metodología CROA.

Fuente: ilustración adaptada de Sanz et al. (2014).

a) En la etapa de Análisis se identifican las necesidades educativas que originan la creación del OA y se propone responder a una serie de preguntas que orientan este análisis.

b) En la etapa de Diseño, se aborda los aspectos de diseño instruccional y de la estructura que tendrá el OA. Se analiza el diseño multimedia, a partir de una serie de escenarios que se proponen por defecto, a manera de guía para los autores.

c) En la etapa de Desarrollo, se debe abordar la selección de recursos (imágenes, videos, documentos de texto, presentaciones) para incorporar en el OA. Luego, se deben utilizar las plantillas elegidas, desarrollarlas en la/s herramienta/s de autor correspondientes, y finalmente integrarlas para conformar el Objeto citado, y empaquetarlo.

d) En la etapa de Publicación se propone publicar el Objeto de Aprendizaje en un repositorio de un Entorno Virtual de E/A. En el caso de nuestro proyecto, tal como se ha explicado, se levantaron sobre la plataforma Moodle de CEDIA.

e) En la etapa de Evaluación se analizan las posibles barreras encontradas en el uso del objeto de aprendizaje en contextos específicos. Hasta la realización de este proyecto, se han encontrado diferentes métodos de evaluación, como la Herramienta de Evaluación de Calidad de OA basada en CodA (Fernández, Domínguez, \& de Armas, 2012).

\section{Fase 6: Pruebas, Implantación y evaluación de resultados}

El curso en-línea de Fundamentos de Seguridad de la Información, está implantado en Moodle de CEDIA, cuya dirección IP es: 190.15.141.97. Este curso está organizado por lecciones o módulos (temas) y por OA (subtemas), 
donde cada objeto se compone por un objetivo de aprendizaje, contenido educativo, actividades de aprendizaje, autoevaluación y metadatos. El primer curso denominado Programas Indeseables o Maliciosos contiene nueve OA y se compone por tres lecciones que son: a) Conceptos Generales del Malware; b) Amenaza de Denegación de Servicios; c) Ingeniería Social. El segundo curso denominado Tipos de Ataques de Ciberseguridad contiene nueve OA y se compone por dos lecciones que son: a) Conceptos Generales de los ataques informáticos; b) Tipos de Ataques. El tercer curso denominado Análisis de Vulnerabilidad contiene ocho OA y se compone por una lección que es: a) Análisis de Vulnerabilidades. Cada curso contiene un grupo de problemas propuestos y resueltos utilizando la técnica didáctica de ABP.

Para corroborar los beneficios del diseño didáctico de cursos en-línea con OA, se aplicó una evaluación en base a la Matriz COdA (Fernández et al., 2012), a dos grupos de 22 y 28 estudiantes respectivamente, del cuarto nivel de la carrera de Ingeniería de Sistemas e Informática que requieren este conocimiento. Este instrumento evaluó los siguientes criterios: i) objetivos y coherencia didáctica; ii) calidad de contenidos; iii) capacidad de generar reflexión, crítica e innovación; iv) interactividad y adaptabilidad del objeto de aprendizaje; v) motivación; vi) formato y diseño; y vii) usabilidad. Luego del procesamiento estadístico, se pudo determinar que se obtuvieron resultados entre 4 y 5 (de una escala ente 1 y 5), para los siete parámetros de evaluación seleccionados, según el siguiente detalle:

a) Los resultados muestran un equilibrio de calidad en los parámetros de evaluación.

b) El parámetro con mayor puntaje es el de Objetivos y coherencia didáctica, esto quiere decir que lo que se aprende con el curso, está de muy acuerdo a los objetivos planteados, los destinatarios sienten que las habilidades adquiridas son las necesarias y las instrucciones de usos están bien definidas.

c) Los parámetros evaluados tanto de carácter didáctico y tecnológico (OA y EVEA) se pudieron valorar de forma conjunta, esto quiero decir que el desarrollo de cursos online implementando ambas metodologías, proporcionan buenos resultados.

Como segundo aspecto de evaluación, se compararon los beneficios del diseño didáctico de cursos en-línea con OA, versus los cursos en-línea tradicionales. Para este propósito, se aplicó una adaptación de la matriz de evaluación de diseño didáctico de cursos virtuales de la Universidad Pontificia Bolivariana. Esta matriz fue aplicada a los mismos grupos de estudiantes que se les aplicó la herramienta COdA. Este instrumento evaluó lo siguiente: i) presentación y contextualización; ii) planteamiento de objetivos; iii) coherencia de contenidos; iv) actividades de aprendizaje; v) evaluaciones, vi) usabilidad e interactividad. Luego del procesamiento estadístico, se pudo determinar que en todos los parámetros evaluados, el diseño de cursos online con OA supera significativamente al diseño de cursos online Tradicionales, lo cual contrasta nuestra hipótesis (figura 3).

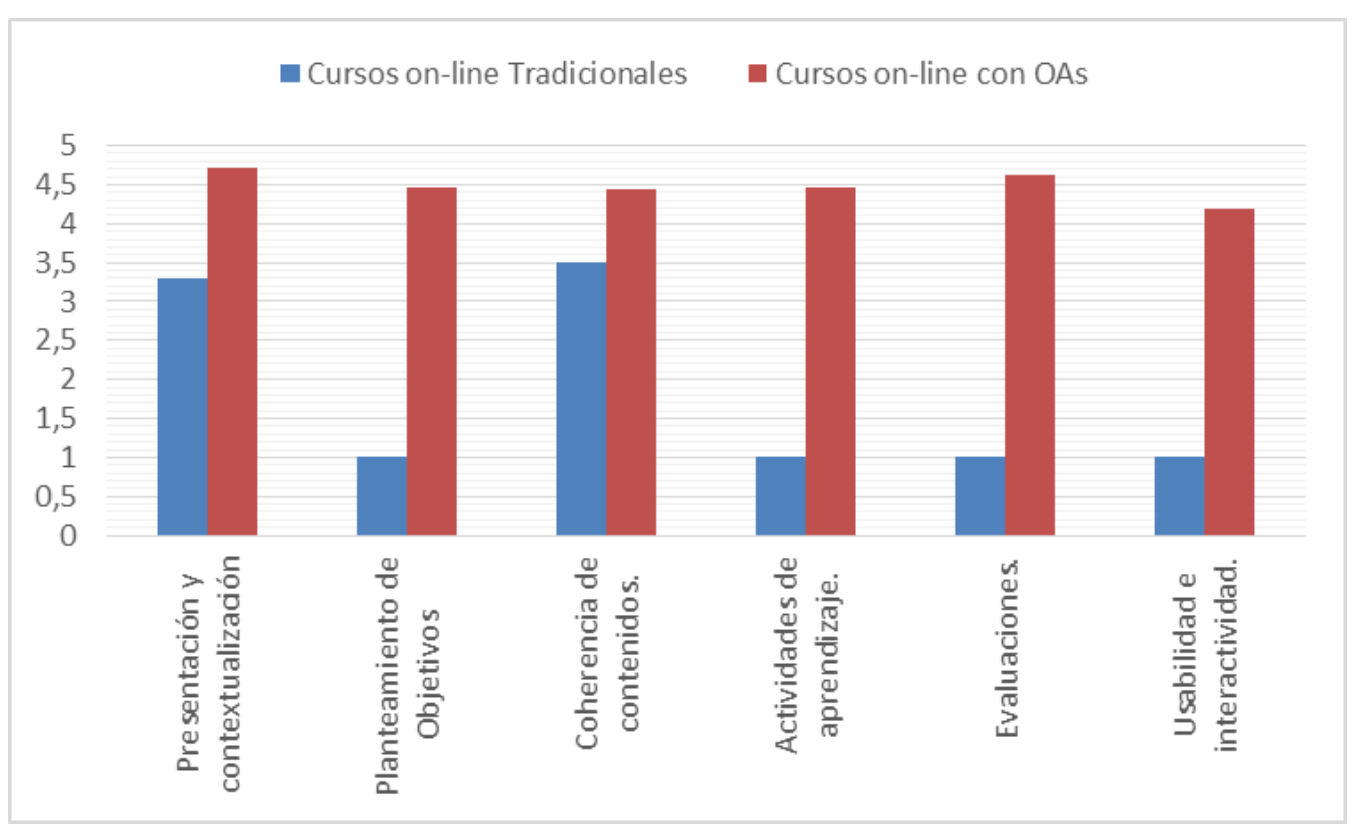

Figura 3. Resultados de la evaluación del diseño didáctico de cursos virtuales on-line. Fuente: los autores.

Finalmente, contrastando con la premisa de investigación de este estudio, se ha demostrado que se cumple los dos primeros niveles de complejidad creciente: conocimiento y comprensión de la taxonomía de Bloom, pues se ha integrado OA, EVEAS con ABP, lo que ha permitido articular los tres ámbitos fundamentales en la educación: enseñanza, aprendizaje (i.e. ámbito cognitivo, afectivo y psicomotor) y evaluación (Bloom, 1956), utilizando tecnología de la era digital. 


\section{CONCLUSIONES}

Este estudio tuvo como objetivo estimular la comprensión conceptual y procedural de estudiantes de carreras técnicas, a través de una propuesta metodológica para el diseño e implementación de cursos en-línea basados en OA. Para ello, se aplicaron OOHDM para el diseño navegacional del entorno virtual de aprendizaje de cursos en línea y el diseño conceptual de la aplicación y la metodología CROA para el diseño de los AO, donde la principal ventaja de su aplicación era lograr una adecuada estructuración y organización de los OA desde un punto de vista pedagógico y tecnológico, sin importar las herramientas de autor que se utilicen para armar los mismos, ni tampoco dependería de los EVEAS donde se suben los OA de un curso. En esta investigación se diseñó, se implementó y se evaluó un curso en-línea de fundamentos de Seguridad de la Información utilizando OA y Moodle. Los resultados muestran la satisfacción de los estudiantes en cuanto a la organización del curso, contenidos, su utilidad, empleo de casos prácticos, uso de medios audiovisuales, utilización de dinámicas de grupo, duración, material empleado, todo con una percepción general favorable desde el punto de vista del diseño instruccional que plantea la metodología CROA. Por lo expuesto, este curso en línea permite estimular la comprensión conceptual y procedural de los estudiantes de carreras de ingeniería. Como trabajo futuro se planea desarrollar una herramienta de autor para crear ambientes virtuales y que agregue automáticamente las bondades de objetos SLOODLE (Simulation Linked Object Oriented Dynamic Learning Environment) open source. 


\section{REFERENCIAS}

Alonso, M., Castillo, I., Martínez, V., \& Muñoz, Y. (2013). MEDOA: Metodología para el Desarrollo de Objetos de Aprendizaje. En $12 a$ Conferencia Iberoamericana en Sistemas, Cibernética e informática: CISCI.

Bloom, B. S. (1956). Taxonomy of educational objectives: the classification of educational goals. Longmans Green and Company.

De Giusti, A., Zangara, A., Sanz, C., Moralejo, L., Barranquero, F., \& Naiouf, M. (2015). Producción de Objetos de Aprendizaje para la enseñanza universitaria. En 21o Congreso Argentino de Ciencias de la Computación.

Fernández, A., Domínguez, E., \& de Armas, I. (2012). Herramienta de evaluación de la calidad de Objetos de Aprendizaje (Herramienta COdA): Guía para la producción y evaluación de materiales didácticos digitales (1.1 ed.). Universidad Complutense de Madrid. (Licencia de Creative Commons: Reconocimiento - No comercial)

García Vera, V. E., Roig Vila, R., Marti Ciriquian, P., \& García Ferrández, P. (2012). Uso de recursos digitales en el ámbito de ingeniería de edificación. En 10a Jornadas de Redes de Investigación en Docencia Universitaria. Universidad de Alicante.

Maldonado, J., Carvallo, J., \& Siguencia, J. (2015). Consorcio Ecuatoriano para el Desarrollo de Internet Avanzado (CEDIA) [Repositorio Digital].

Mestre, G., Torres, E., Díaz, D., \& Gómez, J. (2014). Lineamientos pedagógicos, comunicativos y tecnológicos para la producción de cursos mediados por TICs en la educación a distancia (1a ed.). Cartagena de Indias, Colombia: Universidad Tecnológica de Bolívar.

Pressman, R. S. (2010). Ingeniería del software: un enfoque práctico (6a ed.). México DF, México: Editorial McGraw-Hill.

Sanz, C., Moralejo, L., \& Barranquero, F. (2014). Metodología CROA [Materiales del Curso de Doctorado]. Universidad Nacional de la Plata, Argentina. Descargado de http:// croa.info.unlp.edu.ar

Soto de Giorgis, R., Palma Muñoz, W., \& Roncagliolo de la Horra, S. (2002). Propuesta de un modelo navegacional para el desarrollo de aplicaciones basadas en OOHDM.

Cómo citar este shortpaper (APA):

Zambrano, M. E., Fuertes-Diaz, W. M., Pérez-Herrera, D. D., Villacís, C. J. \& Pérez-Estévez, E. (2016). Producción de cursos en-línea basados en objetos de aprendizaje: una propuesta metodológica orientada a estudiantes de carreras técnicas. AtoZ: novas práticas em informação e conhecimento, 5(2), 115 - 121. Descargado de: http://dx.doi.org/10.5380/atoz.v5i2.49884 